\section{Backside Etching of GaAs Devices}

Jeffrey A. Mittereder

Naval Research Laboratory, Washington, D.C.

The following is a technique for analyzing the area underneath a GaAs integrated circuit or discrete device which may aid in failure analysis. This procedure has been used in the past by the microelectronics community, and it is reviewed here for GaAs monolithic microwave integrated circuits (MMICs) and discrete devices. Because it is a destructive method, we use it in our lab after all other testing is completed. The substrate thickness of the GaAs is $\sim 4$ mils $(25 \mu \mathrm{m})$.

1. First of all, the top side of the field effect transistor (FET) based MMIC is photographed to identify the failure sites, optically or with an SEM, as shown in Figure 1.

2. Next, if the device is die attached by eutectic solder, the circuit is removed from the package by delicately using tweezers while slightly heating the package. Sometimes we have a processing lab do this for us. This procedure is tough for discrete devices due to their small size. We have not attempted this on epoxied devices, although heating may be applicable.

3. The circuit is placed face down on an SEM sample holder, and then cold mounted in epoxy as shown in Figure 2 (a) and (b). The double-sided carbon tape keeps the metallization pattern intact, and also decreases specimen charging while imaging with an SEM.

4. When the cold mount is dry, the Au-Sn back side metal (which is usually silver colored) is sanded off; sanding is stopped when the substrate is reached (usually dark colored). 400 grit sandpaper works fine.

5. The GaAs substrate is then etched using 4 parts hydrogen peroxide, 1 part ammonium hydroxide. The etching progress is checked every 5-10 minutes under an optical microscope. It is stopped when the metallization pattern is revealed and/or the substrate is removed in the area of interest Figure 3.

The top of Figure 3 shows the gate line shorted to the source, a feature which was underneath the air-bridge. The bottom of Figure 3 shows the source shorted to the drain. This view was not visible from the top of the failed circuit - Figure 1. The backside etching technique can most likely be used for silicon devices as well as with a change in the substrate etch (step 5).

Acknowledgements: This work was supported by the Office of Naval Research. The author wishes to thank Dr. Keith Christianson of NRL for helpful discussions.

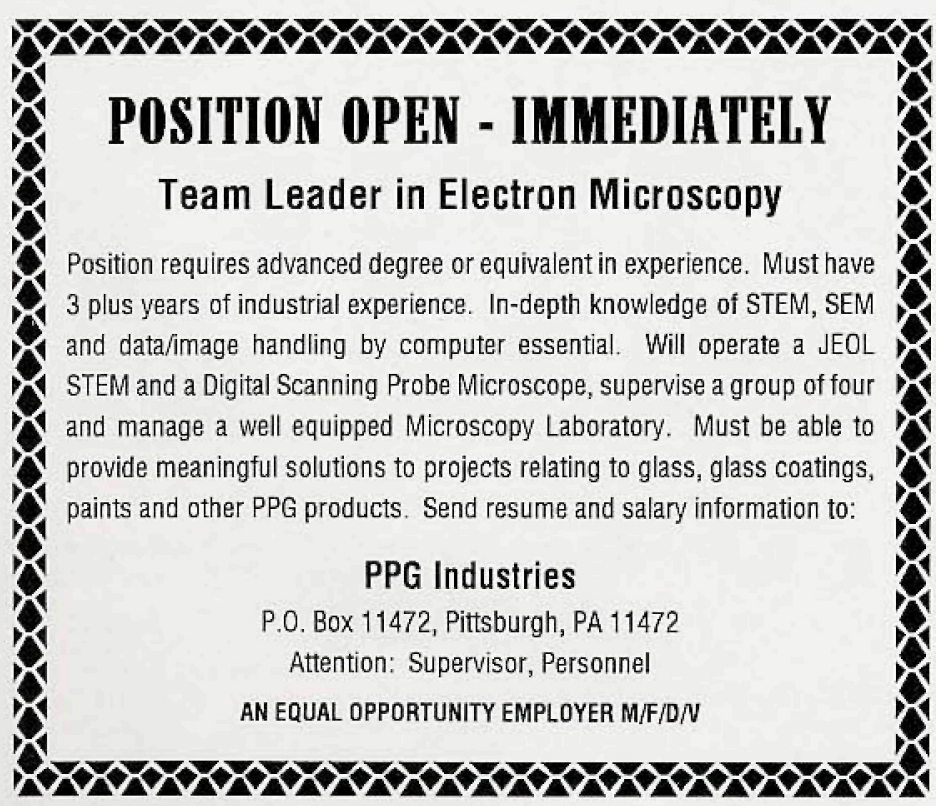

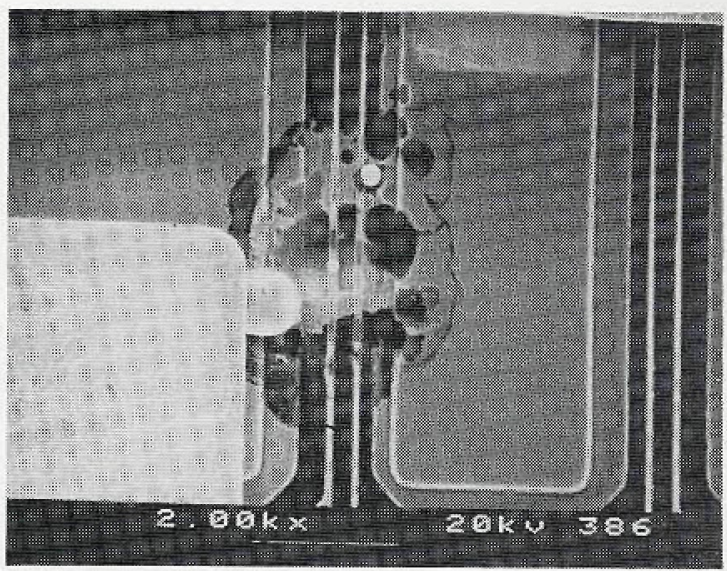

Figure 1: Top View of catastrophic failure site of GaAs MMIC at FET Q3. $\mathrm{Au} / \mathrm{Sn}$
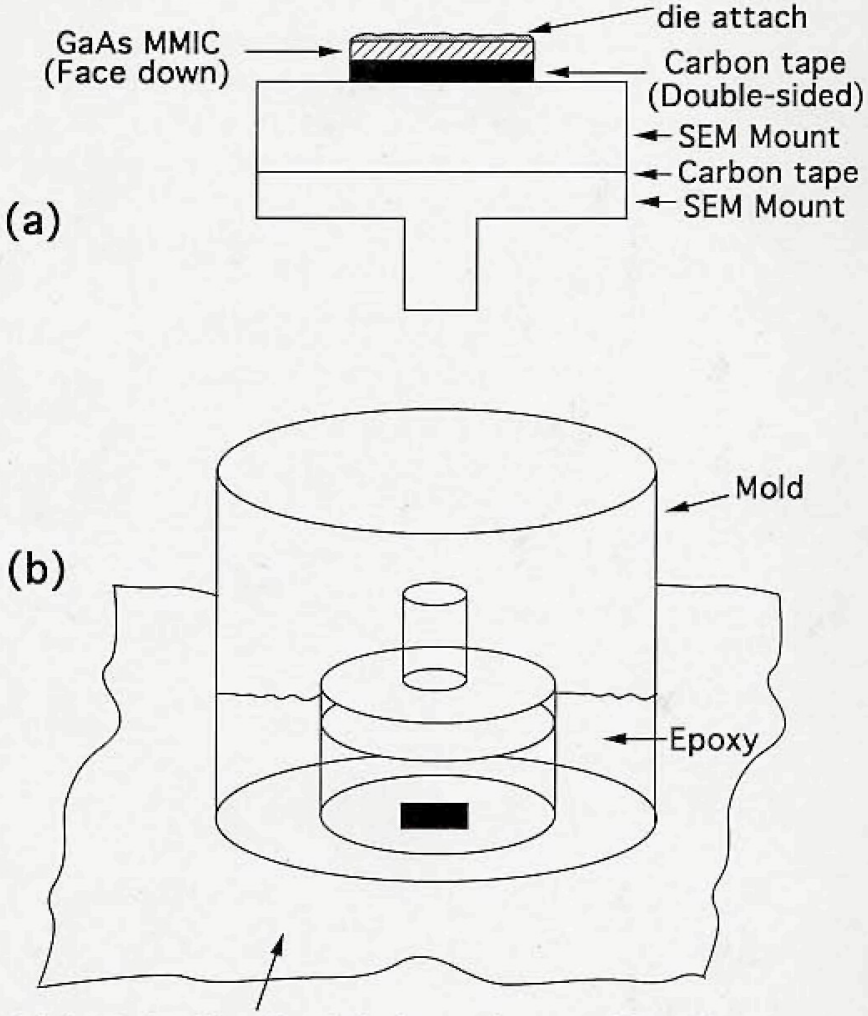

Sticky side of mailing label taped to cardboard

Figure 2: Mounting of GaAs MMIC

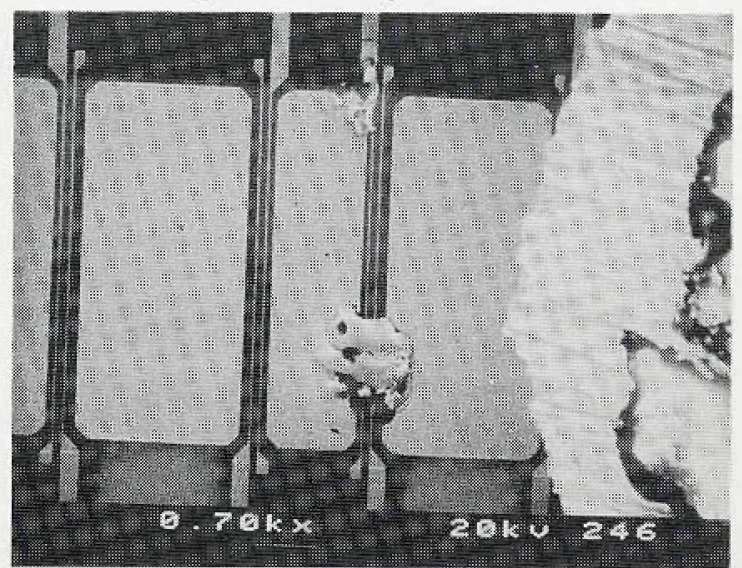

Figure 3: Backside view of failure site from Figure 1. This view reveals features not noticeable in the top view 


\section{You're seeing the clear difference between Hitachi's VP SEM and all the rest.}

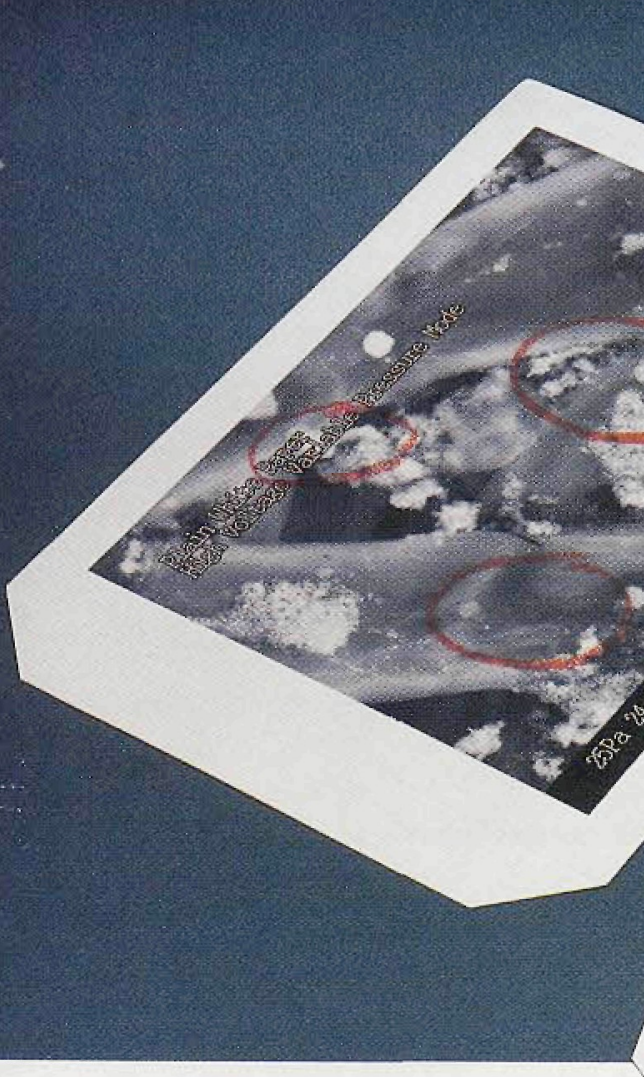

The image on the right was produced by our S-3200N VP SEM-at $5 \mathrm{kV}$. Look at its surface detail. Its resolution. Its brightness. Now compare it with the one on the left, produced at $20 \mathrm{kV}$. You'll see there really is no comparison. And the fact is, you won't find any other VP SEM able to match the S-3200N's performance.

Nor should you expect to. After all, we developed the first VP SEM And this fourth-generation S-3200N simply lengthens our lead. It does so with a new, higherefficiency backscattered electron detector. Plus a unique dual bias circuit that - by increasing emission

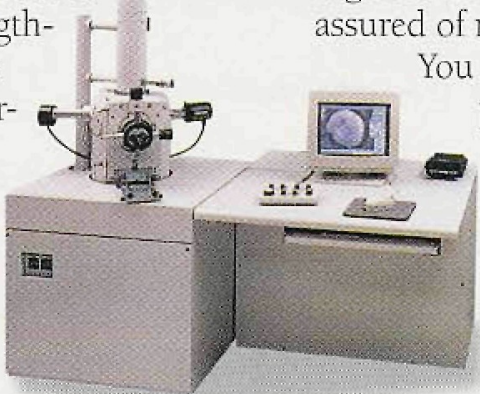

current at low

voltages - results in:

(1) enhanced surface detail, (2) higher resolution, (3) brightness well beyond that possible with $\mathrm{LaB}_{6}$, and (4) a high $\mathrm{X}$-ray count rate.

Obviously, with the $5-3200 \mathrm{~N}$ comes better detection of light elements during EDS and WDS analysis. You're assured of minimized beam damage. You can count on sharp 2048 pixel resolution in digital images. And, with a vacuum system that takes you - at the touch of a button-from atmosphere to low vacuum (.01 to 2 Torr) in about
90 seconds, you can avoid tedious sample preparation and observe specimens in their natural state.

Call, visit our Web site or E-mail us for details on the S-3200N or our S-3200H high-vacuum version. Because, in VP SEMs, the Hitachi difference is clearer than ever.

\section{HITACHI \\ SCIENTIFIC INSTRUMENTS}

Nissei Sangyo America, Ltd.

755 Ravendale Drive

Mountain View, CA 94043 (800) $227-8877$

E-mail: sidsales@nissei.com www.nissei.com

25 West Watkins Mill Road Gaithersburg, MD 20878 (800) 638-4087 